\title{
Neuroendocrinology 1979;28:451-452
}

\section{Author Index}

Alonso, R. 196 Anand Kumar, T.C. 103 Annunziato, L. 435 Arendash, G.W. 349

Bain, J. 425 Baizman, E. R. 402 Banerji, T.K. 201 Barfield, R.J. 217, 228 Barnes, M. 11 Barofsky, A.-L. 358 Baylé, J.D. 114 Beltramino, C. 320 Bennett, G.W. 394 Bllask, D.E. 36 Bornstein, M.B. 131 Boulange, M. 25 Brandt, S.J. 234 Briaud, B. 169, 297, 371, 377 Brown, P. A. 273 Brown, T.S. 123 Burlet, A. 25

Campbell, G.T. 131 Caraceni, T. 313 Cardinali, D.P. 187 Castro-Vazquez, A. 3 Chateau, M. 25 Chi, H.J. 73 Clifton, D.K. 442 Cocchi, D. 313 Cooper, R.L. 234 Collu, R. 178 Cox, B.M. 402 Davidson, J.M. 155 Davis, P.G. 217, 228 Davis, S.L. 256 De|Nicola, A.|F. 64 DiRenzo, G.F. 435 Du Ruisseau, P. 178

Endersby, C.A. 415 Enjalbert, A. 371

Feder, H.H. 92

Feldman, S.C. 131 Frankel, A.I. 302

Gallo, R.V. 349 Gisel, E.G. 212 Goldhaber, G. 44 Goldstein, A. 402

Hayashi, S. 145 Heybach, J.P. 273,329 Hirschmann, N. 44 Hoffman, W.E. 289

Innes, D.L. 212

Jackson, G.L. 256 Jallageas, M. 114 Johnson, A.B. 131 Johnson, L.Y. 36 Johnston, P.G. 155 Jutisz, M. 281

Kachi, T. 201 Kaplan, S.E. 64 Kato, R. 241 Kennaway, D.J. 264 Koch, B. 169, 377 Kochman, K. 248 Kordon, C. 371 Krauss, B. 339 Kuhl, H. 339

Libertun, C. 64 Linnoila, M. 234 Linton, E.A. 394 Lombardi, G. 435 Luborsky-Moore, J. L. 11 Luciani, A. 248 Lutz-Bucher, B. 169, 377

Makino, T. 241 Malven, P.V. 160 Marchetti, J. 25 Marrone, B.L. 92 Mas, M. 196 McCann, S. M. 3 
McDonald, P.|A. 151 Mialhe, C. 169, 297, 371, 377 Mizukami, S. 145 Moos, F. 138 Müller, E.E. 313 Muraki, T. 241 Murphy, H.M. 123

Nair, R.M.G. 11 Nakadate, H. 241 Nir, I. 44

Oaknin, S. 196 Oliver, J. 114 Osman, O.H. 402 Ostrowska, A. 248

Panerai, A.E. 313 Parati, E.A. 313 Peek, J. C. 52 Phillips, M.İ. 289 Polkowska, J. 281 Preziosi, P. 435 Prieto, L. 196

Quay, W.B. 201

Radford, H.M. 307 Reiter, R.J. 36 Réthelyi, M. 82 Richard, Ph. 138 Rodriguez-Sierra, J. F. 92 Ruse, J.L. 425 Ryan, E.L. 302

Sandow, J. 339 Sawyer, C.H. 442 Scapagnini, U. 435 Schettini, G. 435 Schmid, P.G. 289 Schmitt, G. 297 Sole, M.J. 425 Scopacasa, F. 435 Shani, J. 44 Shin, S.H. 73 Stutinsky, F. 297 452 
Author Index

Taché, Y. 178 Taleisnik, S. 320 Taubert, H.-D. 339 Telegdy, G. 386 Thomas, N. 11 Tokunaga, Y. 241 Tóth, É.K. 386 Tulılsi, R.S. 264 
Umberkoman-Wiita, B. 103

Vacas, M.I. 187 Van Loon, G.R. 425 Várszegi, M. 386 Vaughan, M.K. 36 Vermes, I. 386 Vernikos-Danellis, J. 273, 329 
Villier, C. de 11

Walker, R.F. 234 Watkins, W.B. 52 Weet, J.F. 289 Whitehead, S.A. 394 Wideman, C.H. 123 Wilson, C.A. 415 